\title{
Modelos Explicativos da Memória Prospectiva: Uma Revisão Teórica
}

\author{
Patricia Matos ${ }^{1}$ \\ Pedro B. Albuquerque \\ Escola de Psicologia da Universidade de Minho
}

\begin{abstract}
RESUMO - Neste artigo é apresentada uma revisão da literatura sobre os mecanismos cognitivos associados à memória prospectiva, organizados de acordo com a divisão das diferentes fases da recordação prospectiva (i.e., codificação, retenção e recuperação). Inicialmente, é apresentada a diversidade de dados da investigação que sustentam diferentes abordagens explicativas do fenômeno de recuperação de intenções, considerando a natureza automática ou estratégica da memória prospectiva. Em seguida, são salientadas as potenciais explicações sobre os mecanismos presentes durante o intervalo de retenção e na fase de codificação.
\end{abstract}

Palavras-chave: memória prospectiva, recuperação, retenção, codificação

\section{Explanatory Models of Prospective Memory: A Theoretical Review}

\begin{abstract}
This article presents a review of the literature concerning the cognitive mechanisms underlying prospective memory, organized according to the division of the different phases of this phenomenon (i.e., encoding, retention and retrieval). Initially, a variety of research data is presented supporting different explanatory approaches of the phenomenon of intended actions, namely their automatic and strategic processes. Also some potential explanations are presented about the mechanisms in effect during the retention interval and the encoding phase.
\end{abstract}

Keywords: prospective memory, retrieval, retention, encoding

A memória, na sua concepção mais geral, assume um papel fundamental para a espécie humana. Apesar de ser mais comumente relacionada com eventos passados, a memória implica também a formação e a realização de ações futuras. Recentemente, um dos múltiplos sistemas de memória que ajudam a organizar nosso funcionamento, a memória prospectiva (MP), tem sido alvo de grande interesse. A MP diz respeito às atividades do dia-a-dia que devem ser realizadas em um momento futuro apropriado, sem nenhuma instrução que recorde de forma permanente a necessidade da sua realização (McDaniel \& Einstein, 2000; Smith, 2003).

A partir do início da década de 90 do século passado, a literatura começou a valorizar o estudo da MP pelo reconhecimento de sua contribuição decisiva para a adaptação e desenvolvimento cognitivo do ser humano e por estar presente em grande parte das suas atividades quotidianas. Por outro lado, foi-se constatando que falhas na MP são uma das principais queixas de memória apresentadas tanto em contexto clínico, como em contexto normativo, podendo, neste último caso, acarretar graves consequências quando estão implicadas determinadas áreas de atividade (e.g., aviação, medicina, indústria).

A literatura tem analisado a MP nas suas três vertentes do processo mnésico. Ao nível da codificação, os estudos têm incidido na capacidade de planejamento e na implementação de intenções (e.g., Kliegel, Martin, McDaniel, \& Einstein,

1 Endereço para correspondência: EPSI, Escola de Psicologia da Universidade do Minho, Campus de Gualtar, Braga, Portugal. CEP: 4710-057.E-mail: pedro.b.albuquerque@psi.uminho.pt
2002; McDaniel \& Einstein, 2007). No que respeita à retenção, estudam-se, fundamentalmente, os processos que suportam a manutenção da intenção durante intervalos de tempo mais ou menos longos (e.g., Freeman \& Ellis, 2003; Goschke \& Kuhl, 1993) e, por fim, a investigação sobre o processo de recuperação prospectiva tem assumido especial relevância (e.g., Einstein \& McDaniel, 1990; Smith, 2003).

O estudo da MP começou com a condução de investigação seminaturalista que tinha como objetivo ajudar a compreender o funcionamento da recordação prospectiva. Depois dessa fase começaram a ser propostos novos paradigmas, baseados em procedimentos laboratoriais (e.g., paradigma de dupla tarefa), ou seja, situações com maior rigor metodológico.

No entanto, os resultados das investigações são frequentemente discrepantes ou antagônicos e, assim, este artigo tem por objetivo ajudar a clarificar o processamento cognitivo subjacente à recuperação prospectiva, assim como discutir o papel de algumas variáveis que poderão contribuir para a diversidade e antagonismos de alguns dos resultados apresentados. A revisão efetuada baseou-se, majoritariamente, em publicações referenciadas pelas bases de dados PsycInfo e, pontualmente, PubMed e Elsevier Direct.

\section{Definição de memória prospectiva}

A MP é definida como a capacidade de recordar uma ação que se pretende realizar no futuro (intenção), num determinado momento ou local específico, sem nenhuma instrução permanente que nos recorde a realização da 
ação (Einstein \& McDaniel, 1990). Por exemplo, tomar a medicação em momentos específicos ou confirmar previamente procedimentos em cirurgias são exemplos de tarefas de MP. Apesar do debate e de alguma controvérsia acerca da relação entre a MP e a memória retrospetiva (MR), segundo Einstein, Holland, McDaniel e Guynn (1992), na concretização de uma intenção é necessário recordar o momento ou local adequado para a sua realização (componente prospectiva), mas também o seu conteúdo (componente retrospectiva).

A partir de 1985, a proliferação de estudos acerca da MP revelou estarmos perante um tipo de memória com características diferentes não só da MR, mas também das tarefas de vigilância e dos comportamentos habituais. Em primeiro lugar, ao contrário da MR, no desempenho prospectivo não existe nenhuma instrução permanente que nos recorde a necessidade da sua realização (McDaniel \& Einstein, 2000) e, ademais, a intenção deve ser desempenhada num momento específico (Guajardo \& Best, 2000); por outro lado, a recordação prospectiva implica o planejamento das ações, uma vez que estas têm que ser realizadas no decorrer de outras atividades, o que não se verifica na MR (Eisntein \& McDaniel, 1996). Em segundo lugar, as tarefas de MP distinguem-se também das tarefas de vigilância, dado que nestas não é necessário interromper uma atividade para realizar a intenção, mantendo-se, assim, continuamente ativas na memória de trabalho (Graf \& Uttl, 2001). Por último, a MP difere, ainda, dos hábitos, pois estes são apoiados ou por um conjunto de pistas fisiológicas e ambientais, ou por sua prática frequente (Kliegel, McDaniel, \& Einstein, 2008).

Importa referir que o conceito de MP não é um conceito unitário, dado que - como é reconhecido pelos principais investigadores da área - a realização de uma intenção pressupõe várias etapas, que implicam diferentes modalidades ou estratégias de processamento e que não depende exclusivamente da memória, pois necessitam de capacidades executivas como as atencionais. No sentido de compreender a forma como esses processos de caráter executivo se encontram envolvidos nesse tipo de memória, vários autores têm proposto diferentes etapas e características na recordação prospectiva. Recentemente, McDaniel e Einstein (2000) apresentam uma sequência de quatro fases envolvidas na recordação prospectiva: a) a intenção é formada (codificação/planeamento da intenção); b) ocorre um intervalo de tempo durante o qual o sujeito está ocupado com outra atividade e a intenção é retida (retenção da intenção); c) após este intervalo de tempo, a intenção é recuperada por iniciativa própria perante pistas internas ou externas; e, por fim, d) a intenção é realizada de acordo com o plano (execução da intenção).

É sabido que as tarefas prospectivas requerem uma resposta a pistas, internas e externas, associadas a intenções. As pistas externas são explícitas e encontram-se no ambiente (e.g., ver o caixa eletrônico multibanco e lembrar-se de pagar a conta de telefone) ou são referidas por alguém (e.g., “já tomaste a medicação?"), enquanto as pistas internas são geradas pela própria pessoa, ou seja, emergem sem esforço

Tabela 1.Tipologia de tarefas de memória prospetiva (adaptado de Dismukes, 2010).

\begin{tabular}{|c|c|}
\hline Tipos de tarefas & Exemplos \\
\hline Baseadas nos eventos & Dar um recado a um amigo quando o encontrarmos \\
\hline Baseadas no tempo & Retirar o bolo do forno após 20 minutos \\
\hline Baseadas no tempo & Ir a uma consulta médica às 10:00 horas \\
\hline Atenção dividida & Conduzir e utilizar o telemóvel simultaneamente \\
\hline Tarefas sequenciais de uma atividade de rotina & Procedimentos de colocar um avião em funcionamento \\
\hline Substituição de uma atividade por uma atividade de rotina & $\begin{array}{l}\text { Desvio do trajeto para o percurso habitual para o local de } \\
\text { trabalho }\end{array}$ \\
\hline Interrupções & Interromper uma apresentação para realizar um telefonema \\
\hline
\end{tabular}

consciente e, por dependerem de um impulso interno, são menos controláveis.

\section{Tipos de tarefas de memória prospectiva}

A tipologia mais aceita para a distinção das tarefas de memória prospectiva foi proposta por Einstein e McDaniel (1990) e distingue as tarefas baseadas no tempo, nas quais o participante tem de recordar e executar uma ação em um momento específico (i.e., a uma determinada hora) ou após ter passado um determinado tempo concreto (e.g., retirar o bolo do forno após 20 minutos), e as tarefas baseadas em eventos ou acontecimentos, nas quais o participante deve realizar uma ação quando se dá a ocorrência de determinado acontecimento alvo, frequentemente uma pista (e.g., dar um recado a um amigo quando o encontrarmos).

Outros autores (Ward, Shum, McKinlay, Baker-Tweney, \& Wallace, 2005) apresentam outras formas de categorização, distinguindo as intenções de curto prazo, que devem ser 
concluídas em alguns minutos, das intenções de longo prazo, cuja realização pode ocorrer passadas várias horas. Ellis (1996) apresentou também uma divisão das tarefas baseadas no tempo: tarefas esporádicas, que devem ser realizadas em um momento particular; e tarefas encadeadas, que implicam uma realização prolongada no tempo.

Na sequência da classificação de Meacham e Leiman (1982), que distinguem as tarefas habituais das tarefas episódicas, Loukopoulos, Dismukes e Barshi (2009) subdividem essas últimas tarefas. Assim, acrescentam a distinção entre as tarefas em que ocorrem interrupções e é necessário que o sujeito faça um resumo da atividade anterior para continuar a realizar a tarefa de MP e as tarefas que necessitam de atenção dividida nas atividades decorrentes - as quais pretendem simular, em contexto laboratorial, o envolvimento real do participante numa tarefa no momento em que surge uma pista de MP no contexto. Dismukes (2010) enfatiza também a diferença entre as tarefas sequenciais e as atividades de rotina, assim como a substituição de uma atividade não-típica por uma atividade habitual. A Tabela 1 apresenta, de forma esquemática, os vários tipos de MP apresentados pelos autores.

Tendo em consideração as características naturais das tarefas de MP, Ellis e Kvavilashvili (2000) recomendam que, para os procedimentos laboratoriais terem validade, devem ser considerados três aspetos fundamentais: a) um intervalo de retenção entre a formação da intenção (codificação) e o momento da realização da intenção (recordação); b) a ausência de uma instrução específica que indique o momento apropriado para realização da ação; e c) a necessidade de interromper uma atividade decorrente para realizar a ação.

Com o intuito de fornecer uma descrição organizada dos resultados dos diversos estudos realizados, foram consideradas as três fases envolvidas na MP e que compõem o procedimento mnésico: codificação, retenção e recuperação da informação. Para que seja mais fácil a compreensão total dos procedimentos e resultados nos estudos, será abordado, inicialmente, o processo de recuperação das tarefas de MP.

\section{Recuperação da memória prospectiva}

A recuperação da memória prospectiva assumiu destaque enquanto domínio da investigação e um dos aspetos mais relevantes diz respeito à discussão acerca do seu caráter estratégico ou automático, discussão que tem salientado duas visões antagônicas da recuperação de intenções. A primeira pressupõe que a recordação prospectiva se deve a um processo voluntário e estratégico (e.g., Harris, 1984). Essa visão apresenta alguns problemas porque exige a utilização de recursos atencionais nas tarefas prostetivas, o que tornaria o processo cognitivo mais complexo e com elevado prejuízo para a realização de outras atividades cotidianas simultâneas. A alternativa consiste em um modelo que defende uma recuperação automática das tarefas de MP (Einstein \& McDaniel, 1990). Mais tarde surge a abordagem multiprocessual (McDaniel \& Einstein, 2000), que apoia a recordação automática, mas que reconhece também o uso de capacidades conscientes na recordação prospectiva em certas atividades e contextos, e o modelo pré-frontal (Martin, Kliegel, \& McDaniel, 2003).

A primeira visão assume claramente o envolvimento de recursos atencionais responsáveis por uma monitorização estratégica do ambiente em busca das pistas de MP ou por periodicamente trazerem à memória de trabalho a intenção de realização prospectiva, e considera que a concretização dessas tarefas prejudica o desempenho na atividade decorrente. Os recursos especificamente associados ao Sistema de Supervisão (SAS; cf. Norman \& Shallice, 1983) estariam envolvidos na associação entre a pista e a intenção na fase de codificação, seriam responsáveis pela monitorização do ambiente e, por fim, permitiriam também interromper a atividade decorrente quando surge o momento indicado para realizar a ação. Essa ideia foi inicialmente testada por Harris, em 1984. Em seu estudo, com recurso ao procedimento Test-Wait-Test-Exit, o autor pretendia evidenciar que, devido ao elevado custo atencional de uma monitorização constante nas atividades do dia-a-dia, o sujeito monitoriza de forma periódica se as condições ambientais são apropriadas para a realização de uma intenção baseada no tempo. Desse modo, o autor pôde constatar que a eficácia da MP está associada à monitorização da intenção e, especificamente, ao aumento dessa estratégia à medida que se aproxima o momento indicado para realizar a ação. A ideia geral que se extrai desse estudo é a de que as pessoas utilizam um processo de monitorização estratégica para cumprir suas intenções. Contudo, esses resultados não permitem explicar, cabalmente, quais são os mecanismos de ativação da monitorização.

Nesse sentido, e no que concerne às tarefas baseadas em eventos, outros autores (Smith \& Bayen, 2004) propõem que o desempenho prospectivo requer um sistema executivo atencional exigente em termos de recursos, capaz de monitorizar continuamente o ambiente em busca de pistas ou ativar conscientemente a intenção na memória de trabalho. Essa proposta permitiria explicar os resultados do estudo de Marsh e Hicks (1998), segundo os quais a elevada exigência atencional das tarefas decorrentes diminui o desempenho prospectivo. Por outro lado, as investigações apontam também para uma redução dos recursos disponíveis para a realização da atividade decorrente, quando o participante sabe que nessa atividade está inserida uma tarefa prospectiva (Smith \& Bayen, 2004), corroborando a ideia de que os processos atencionais preparatórios ocorrem previamente ao aparecimento da pista de MP.

Em uma outra perspectiva, Marsh, Hicks e Cook (2006) consideram que, ao invés de uma monitorização ambiental constante, ocorreria uma monitorização estratégica das pistas contextuais pertinentes para a realização da intenção. No seu artigo, os autores pretendiam saber se a informação prévia dada aos participantes, acerca da fase da experiência em que seriam apresentadas as tarefas de MP, teria influência no desempenho prospectivo. As conclusões do estudo foram as seguintes: quando os sujeitos são informados acerca da fase da experiência em que irá decorrer a tarefa de MP, usam essa informação como pista contextual e restringem a monitorização para esse contexto, o que acarreta custos atencionais para a tarefa decorrente de decisão lexical solicitada nessa fase, em comparação com as outras fases. Esse conjunto de dados parece sugerir, assim, que a 
explicação para a falha de MP estaria associada ao nível de envolvimento dos recursos de atenção do sistema executivo central em outras tarefas, e não seria devida à memória de trabalho (McDaniel \& Einstein, 2007).

Nem todos os estudos corroboram a hipótese estratégica e, como referimos anteriormente, uma outra linha de investigação defende a importância dos processos de recuperação espontânea da intenção. Para estudá-los, Einstein e McDaniel (1990) conceberam um procedimento experimental baseado no paradigma de dupla tarefa. O tempo de realização da atividade decorrente vem sendo investigado como potencial fator de diferenciação dos modelos teóricos apresentados. A observação de que em determinadas situações não há um prejuízo na realização da atividade decorrente (e.g., quando a tarefa de MP é simples ou as pistas são salientes), tem levado alguns autores a questionar a monitorização estratégica no processo de recuperação prospetiva e a hipotetizar a ocorrência de um processo mais automático. A título de exemplo, os resultados da experiência de Einstein et al. (2005, Experimento 1) revelam que a tarefa de MP pode ser realizada sem causar interferência na atividade decorrente, quando os sujeitos têm apenas uma tarefa de MP a cumprir, o que pressupõe uma recuperação automática.

De forma geral, o processo de recuperação automática da MP é baseado em várias linhas de evidência: 1) o desempenho na atividade decorrente não é comprometido pela tarefa de MP, 2) quando existe uma associação entre a pista e a intenção não é necessário o recurso a um processo de monitorização e, por último, 3) mesmo na presença de uma atividade decorrente atencionalmente exigente, o desempenho prospectivo não é afetado. Por tudo isso, é de salientar que a hipótese de um processo mais automático contribui para a progressiva validação na caracterização da recuperação prospectiva.

Em síntese, os modelos explicativos da MP apresentados diferem entre si: para uns, o envolvimento de um sistema atencional supervisor conduz a que a recuperação prospetiva dependa de ensaios autoiniciados e da monitorização estratégica do ambiente; para outros, a MP tem por base processos automáticos apoiados por um processamento independente do contexto e baseado na familiaridade ou em processos de atribuição de discrepâncias.

No entanto, há pelo menos duas linhas de evidência que não suportam totalmente as abordagens teóricas anteriores: por um lado, o declínio no desempenho prospectivo verificado com o avanço da idade, de acordo com a suposição de que há um déficit atencional que aumenta com a idade e que interfere, assim, na realização das tarefas de MP; e, por outro lado, a evidência de que não há grandes diferenças entre os adultos jovens e idosos na recordação prospetiva em tarefas baseadas em eventos, o que é coerente com a abordagem automática (e.g., Einstein \& McDaniel, 1990; Einstein et al., 1992). McDaniel e Eisntein (2000) propuseram, assim, uma perspectiva integradora, a abordagem multiprocessual. De acordo com esta perspectiva 1) verifica-se uma tendência para o sujeito se apoiar em processos automáticos de recuperação das intenções, mas 2) as pessoas são capazes de recorrer a diferentes processos para realizar diferentes tarefas de MP e, por fim, 3) o processo utilizado e a sua eficácia dependem de vários aspetos, tais como das características da tarefa de $\mathrm{MP}$, da natureza e exigência da atividade decorrente e das características do próprio indivíduo.

Dessa abordagem multiprocessual resultam duas implicações. A primeira é a de que é adaptativo para o sujeito ter um sistema flexível para a realização de intenções, por meio de diferentes mecanismos, para que o desempenho das atividades do dia-a-dia não seja comprometido (McDaniel \& Einstein, 2007). Apesar de os indivíduos apresentarem uma tendência para apoiar-se em processos automáticos de recuperação prospetiva, ocorrendo o pop-up da intenção, em condições particulares torna-se necessário adotar um modo de operação mais estratégico, tal como o planejamento e a repetição periódica da intenção, ou a monitorização do tempo ou do ambiente na procura da pista (McDaniel \& Einstein, 2000). A segunda implicação é a de que o sujeito desenvolve flexivelmente uma estratégia apropriada a cada situação depois de analisar a exigência da tarefa de MP. Nessa linha de pensamento, por exemplo, quando a tarefa de MP implica um longo intervalo de retenção é mais provável que o sujeito adote uma estratégia de monitorização; pelo contrário, se o sujeito antevir encontrar pistas ambientais salientes pode recorrer a um processo de recuperação mais automático (McDaniel \& Einstein, 2007).

De acordo com Kliegel et al. (2002), essa interpretação tem como pressupostos de validação que a recuperação da intenção ocorre sem custos para a atividade decorrente e, portanto, com recurso a um processo espontâneo, quando: a) a ação prospetiva é simples; b) a pista e a ação estão suficientemente associados no momento da codificação; c) a atividade decorrente requer o processamento das dimensões relevantes da pista (processamento focal), e d) a pista é saliente. A contrastar com o bom desempenho na atividade decorrente obtido através das condições descritas acima, assiste-se a um desempenho pobre nesse tipo de atividades quando essas condições não estão presentes (e.g., Brandimonte \& Passolunghi, 1994; Einstein \& McDaniel, 2005; Marsh, Hicks, \& Cook, 2005). Com efeito, a investigação acerca da interferência negativa de tarefas de atenção dividida na MP (Einstein, McDaniel, Smith, \& Shaw 1998; McDaniel \& Einstein, 2000), principalmente quando são atencionalmente exigentes (Marsh \& Hicks, 1998), tem sido a base de evidência de um processo de monitorização.

Para além dos modelos anteriores existe outra perspectiva sobre a MP (e.g., Martin et al., 2003). O modelo pré-frontal de MP foi proposto por West (1996) após a observação da deterioração do córtex pré-frontal em idosos, assim como um desempenho pobre destes indivíduos comparativamente com jovens adultos, em tarefas de MP com grande exigência de funcionamento do lobo frontal. De acordo com esse modelo, as falhas de MP ocorrem devido à redução da eficiência das funções executivas que são mediadas pelo córtex pré-frontal, como por exemplo, a memória de trabalho, o planejamento, a monitorização, o controle inibitório e a alocação de recursos atencionais.

Em síntese, a história da investigação da MP é marcada por duas linhas de interpretação: uma maneira de compreender o modo das pessoas realizarem a recordação prospetiva é assumir que existe um sistema de atenção executiva que conscientemente monitoriza o meio ambiente, 
procurando pistas que iniciem a recordação de intenção; outro modo é considerar que o sistema cognitivo responde automaticamente à ocorrência de pistas ambientais. Ambas as abordagens estão bem sustentadas empiricamente, o que leva alguns autores a considerar a existência de múltiplos mecanismos para a recuperação na MP - abordagem multiprocessual.

\section{Retenção na memória prospectiva}

Um dos temas de maior interesse no estudo da MP refere-se à compressão do que acontece durante o intervalo que medeia a intenção de realizar uma ação no futuro e a sua concretização. Segundo McDaniel e Einstein (2007), as intenções seriam retidas ou mantidas na memória e atingiriam um maior nível de ativação comparativamente a outros conteúdos mnésicos. Os autores pressupõem que, em um contínuo de ativação, as tarefas de MP encontram-se em um nível intermédio, revelando uma maior sensibilidade à deteção de pistas ambientais, o que faz com que sejam mais facilmente ativadas do que outros conteúdos; os autores também defendem que o elevado nível de ativação das intenções seria responsável pelo retrieval mode durante o intervalo de retenção. O retrieval mode é outro tipo de monitorização que permite a recordação prospetiva para além da procura ativa de pistas ambientais, sugerindo-se que após a codificação de uma intenção, o sistema cognitivo está alerta para processar os estímulos como possíveis pistas para realizar a intenção (Guynn, 2003).

Essas considerações podem ser explicadas a partir do efeito de superioridade da intenção (Goschke \& Kuhl, 1993), que postula que os aspetos relacionados com uma intenção adiada (e.g., comprar pão), para que seja realizada num momento mais adequado, parecem mais salientes. Durante esse período, a intenção é armazenada na memória como uma representação verbal explícita. Isto é, os diversos conceitos verbais que se relacionam com essa intenção - como as noções de compras ou comida - são altamente ativos. Como consequência, qualquer aspeto que esteja relacionado com a intenção (de acordo com o exemplo, a palavra maçã) é mais saliente ou proeminente. Um dos primeiros estudos sobre o efeito de superioridade da intenção foi conduzido por Goschke e Kuhl (1993) e replicado em experiências posteriores com tarefas de decisão lexical. Nesse novo paradigma experimental, os autores pediram aos participantes que estudassem dois scripts (sequências de ações organizadas espacial e temporalmente, propostas por Schank \& Aberlson, 1977), manipulando a tarefa final a realizar que poderia ser uma tarefa de reconhecimento ou a realização de um dos scripts. O exemplo típico de um script corresponde a uma ida a um restaurante que inclui determinadas ações como, por exemplo, entrar, sentar-se, ler o menu, fazer o pedido, receber a comida, comer, pedir a conta, pagar e sair. Os resultados obtidos explicam o efeito de superioridade da intenção ao demonstrarem uma maior eficácia na tarefa de reconhecimento relacionada com o script a executar, refletida no menor tempo de execução da tarefa. Os dados sugerem que a intenção de realizar determinadas ações permite a ativação de alguns termos e conceitos que correspondem a essa sequência de comportamentos (Goschke \& Kuhl, 1993).

O elevado estado de ativação das tarefas de MP leva à consideração de que, ao contrário do que tende a acontecer na memória retrospetiva, a retenção de intenções não decai rapidamente à medida que decorre tempo sobre a codificação inicial da informação, em comparação a outros conteúdos mnésicos. Os estudos levados a cabo nesse domínio não apresentam resultados concordantes, pois enquanto uns mostram que o intervalo de retenção está associado a um declínio na MP (Brandimonte \& Passolunghi, 1994), outros não evidenciam este efeito (Einstein et al., 1992; Guynn, McDaniel, \& Einstein, 1998). Outros estudos mostram o contrário: um melhor desempenho prospectivo no caso de um intervalo de retenção mais prolongado. No estudo de Hicks, Marsh e Russel (2000), 19\% dos participantes obtiveram sucesso na tarefa de MP na condição com um curto intervalo de retenção, em comparação com $36 \%$ de recordação prospetiva na condição com um maior intervalo de retenção. Uma das explicações desse fenômeno repousa na concepção, presente na literatura acerca da MR, de que durante o intervalo de retenção ocorreria uma repetição de manutenção (i.e., a ativação da intenção da memória a longo-prazo), a qual permite evitar o esquecimento (Roediger \& Karpicke, 2006). Nesse domínio, seria interessante compreender se no intervalo de retenção, poderá também ocorrer o processo de repetição de elaboração, em que novas associações e significados são acrescentados ao material a processar para que seja facilitada a sua recuperação, à semelhança do que acontece na MR (Craik \& Lockhart, 1972).

Sabendo que um maior intervalo de retenção parece permitir a repetição da recordação da intenção um maior número de vezes e, em consequência, apoiar um bom desempenho prospectivo, importa conhecer os mecanismos que promovem essa repetição. Depois da codificação, a intenção é verificada ocasionalmente e, nesse contexto, é possível distinguir-se dois tipos de explicação: a primeira aponta para uma recordação periódica apoiada por mecanismos internos autoiniciados (Hicks et al., 2000), enquanto uma segunda abordagem salienta o papel das pistas externas na recuperação automática da intenção (Sellen, Louie, Harris, \& Wilkins, 1997). Por exemplo, algumas pistas ambientais podem originar a recuperação automática da intenção de telefonar a um amigo no fim de semana seguinte, como sejam ouvir um celular a tocar ou encontrar o nome da cidade onde o amigo vive.

Consideremos ainda o seguinte: alguns autores fizeram notar que a eficácia do desempenho prospectivo é melhorada não só por meio da recuperação periódica das intenções nos momentos de intervalo (Hicks et al., 2000), mas também quando a recuperação de intenções fica próxima do momento indicado para a sua realização. O estudo de Finstad, Bink, McDaniel e Einstein (2006, Experiência 1) introduziu uma inovação ao incluir pequenas pausas na atividade decorrente, durante as quais metades dos participantes recebia a instrução "Lembre-se do que deve fazer quando vir a palavra-chave" e a outra metade não tinha nenhuma tarefa a cumprir durante esses períodos de tempo. Os participantes do grupo que recebeu instruções para recuperar a tarefa de MP nos 
intervalos demonstraram um melhor desempenho prospectivo (75\%), comparativamente ao outro grupo (60\%).

A aplicação prática do pressuposto de que a solicitação da recuperação de intenções em intervalos programados tem influência no desempenho prospectivo apresenta resultados interessantes. Realçamos como nota distintiva o estudo de Camp, Foss, Stevens e O’Hanlon (1996), o qual buscava conceber programas de reabilitação no âmbito da MP. O estudo foi desenvolvido junto de doentes de Alzheimer, por meio de um método de recuperação de intenções programado para ajudar os participantes a cumprir objetivos diários definidos em um calendário. Os resultados desse programa experimental evidenciam que $87 \%$ dos 23 doentes de Alzheimer, com uma média de 10 sessões de treino, conseguiram referir ao experimentador a sua tarefa de verificar no calendário os seus objetivos diários. Os autores apontam que é provável que a aplicação dessa técnica obtenha sucesso em outros tipos de patologias com déficits no desempenho prospectivo. Esses estudos constituem uma linha de investigação que é urgente desenvolver.

\section{Codificação na memória prospectiva}

Até agora foi discutido o que ocorre na fase de recuperação e de retenção da MP. Embora tenha sido desenvolvida menos investigação relativa à fase de codificação, parece existir consenso relativamente à importância do planejamento na MP, o qual varia em função da complexidade e da importância da tarefa de MP, bem como de algumas características individuais (e.g., Dobbs \& Rule, 1987; Shallice \& Burgess, 1991). Por exemplo, algumas tarefas de MP envolvem várias etapas, realizadas cumulativamente (e.g., escrever e enviar uma carta a um familiar), assim como implicam decisões difíceis (e.g., aceitar um jantar com um amigo e replanejar as tarefas familiares agendadas para essa noite) e diferem na sua importância (e.g., ir buscar um familiar no aeroporto ou comprar pão no caminho para casa).

$\mathrm{Na}$ tentativa de explicação do pressuposto de que o planejamento é importante na fase de codificação, Marsh et al. (2006) mostraram que, quando os participantes são informados da fase em que ocorrerá a tarefa de MP, ocorre interferência na realização da atividade decorrente apenas nessa fase, o que é sugestivo da elaboração de planos por parte dos sujeitos para realizarem a tarefa de MP.

Uma das formas mais aceitas para a determinação da existência de planejamento de intenções na MP resulta dos estudos com o paradigma da tarefa dos seis-elementos (Kliegel et al., 2002). Essa tarefa baseia-se na necessidade de planejamento de várias tarefas, criando uma situação análoga à que vivemos no nosso cotidiano. Assim, em um dos estudos mais importantes nesse domínio, os participantes tinham que cumprir sequencialmente, e em um tempo definido, um total de seis tarefas, organizadas em três conjuntos de duas tarefas. O desempenho nesse tipo de tarefa reflete a capacidade individual de planejamento e de execução, assim como de formação de intenções. Kliegel et al. (2002) estudaram uma amostra constituída por adultos e idosos que receberam instruções acerca da tarefa dos seiselementos. Foi-lhes também dito que deviam iniciar essa tarefa após o preenchimento de um questionário (pista de MP externa). Os dados coletados basearam-se na qualidade dos planos preparados pelos participantes, na capacidade de memorização dos planos elaborados (os sujeitos realizaram uma tarefa distrativa durante 25 minutos e foram depois submetidos a uma tarefa de evocação), na capacidade de iniciarem a tarefa dos seis-elementos e na concordância entre o plano elaborado na fase inicial e o demonstrado na tarefa dos seis elementos.

Os autores concluíram que 1) os participantes adultos e idosos apresentam uma boa memória dos planos elaborados; 2) apenas $50 \%$ dos aspectos planejados foram aplicados na realização da tarefa MP; 3) os adultos mais jovens desenvolveram planos mais complexos do que os idosos; e a 4) maior complexidade dos planos levou a melhores desempenhos prospectivos. Um estudo posterior ajudou a compreender a relação entre a qualidade do planejamento e o sucesso das tarefas de MP. No estudo de Kliegel, Martin, McDaniel, Einstein e Moor (2007), um grupo de participantes (adultos jovens e idosos) recebeu indicações externas para a formulação de planos na tarefa dos seis elementos, nomeadamente, a consideração do questionário como pista externa para a realização da intenção e a necessidade de planejar a mudança para a próxima atividade após completarem duas tarefas da mesma atividade; o outro grupo não recebeu essas indicações. De forma geral, os dados das investigações supracitadas salientam a importância do planejamento na melhoria do desempenho prospectivo, embora esse plano nem sempre seja totalmente utilizado na execução das intenções, à semelhança do que acontece na vida real; mas também ressaltam a importância de um planejamento específico e não baseado em pistas gerais. A formulação de planos na recordação prospectiva parece revelar-se uma técnica promissora a integrar em programas de intervenção, ainda que subsistam algumas limitações.

Todavia, apesar dos estudos serem limitados, serão apresentadas evidências de investigações que suportam direta e indiretamente a importância de um planejamento específico. Em primeiro lugar, é interessante notar que, nos procedimentos laboratoriais típicos de MP, as instruções com pistas específicas assumem a forma de uma implementação de intenção, pois associam diretamente uma pista a uma ação específica (e.g., pressionar a tecla " $X$ " quando surgir a palavra dormitório). Gollwitzer (1999) sugere que a própria pista ambiental está também num estado de maior ativação e não apenas a tarefa de MP, segundo o efeito de superioridade da intenção. Relativamente à segunda premissa, os dados de algumas experiências são consistentes com a ideia de uma recuperação automática das tarefas de MP após o processo de implementação de intenções. Especificamente, alguns estudos apontam uma melhoria no desempenho prospectivo em adultos com déficit de memória de trabalho após a utilização dessa técnica, com o pedido de informação adicional sobre a tarefa a cumprir (e.g., imaginar os eventos que antecedem a intenção - Liu \& Park, 2004). Nesse sentido, interessa considerar os dados fornecidos por investigações cujas instruções assumem a forma de uma implementação de intenções. No estudo de McDaniel, Guynn, Einstein e Breneiser (2004), os participantes foram sujeitos a uma atividade decorrente de elevada exigência atencional. 
Nesse estudo, as instruções das duas condições atencionais manipuladas permitem a implementação de uma intenção - na condição de elevada associação entre a pista-intenção, quando é apresentada a palavra espaguete, o sujeito deve escrever molho, e na condição de fraca associação, o sujeito deve escrever a palavra campanário. Contudo, os resultados mostram que um bom desempenho prospectivo é alcançado apenas na condição de forte associação pista-intenção. Assim, se esses resultados forem considerados, juntamente com os de Liu e Park (2004), que usam formação adicional nos procedimentos de implementação de intenções, sugere-se que esse processo não permite por si só a articulação pistaintenção que favorece a recuperação automática. Veja-se que, no estudo de Zimmerman e Meier (2010) verificou-se um melhor desempenho prospectivo, principalmente nos idosos, na situação de implementação de intenções. A explicação teórica sobre a efetividade da implementação de intenções baseia-se, assim, no fato de esta não implicar recursos cognitivos e ocorrer de forma mais automática. De fato, essa técnica estabelece a base para a associação, mas parece necessário um processamento adicional ou experiência prévia na situação e esta é uma das vias de estudo em perspectiva.

\section{Conclusões}

Este artigo pretendeu rever e comentar alguns dos resultados mais significativos da investigação sobre memória prospectiva. Isso foi feito tendo em conta duas linhas orientadoras. A primeira centrada na importância desses resultados para a clarificação do tipo de processos envolvidos na MP. E, a segunda, tendo como fio condutor as três fases ou processos da memória: codificação, retenção e recuperação.

Pode-se considerar que as perspectivas conceptuais apresentadas diferem entre si no caráter mais estratégico ou automático que atribuem ao processo de recuperação da informação prospectiva. Ademais, mais do que teorias com corpo estruturado e integrador, parece que estamos perante um conjunto de modelos cuja validação se faz, ou vem sendo feita, a partir da investigação experimental em contexto laboratorial. Essa opção tem ganhos óbvios quanto ao controle metodológico, mas é atacada por se afastar do que "verdadeiramente" acontece quando qualquer um de nós tem que realizar uma ação ou intenção. Sabemos que, para a psicologia da memória, essa é uma questão antiga (Banaji \& Crowder, 1989).

Nesse sentido, o avanço da investigação da MP requer que se estude com maior clareza outros tipos de tarefas de MP (e.g, tarefas baseadas no tempo, tarefas habituais de MP, interrupções, monitorização de tarefas concorrentes), por meio de procedimentos com intervalos de retenção mais longos, em situações mais exigentes e complexas, com múltiplas intenções e objetivos a cumprir, em que o sujeito que tem que reorganizar as suas prioridades face a novas exigências ou oportunidades, à semelhança da vida real. Da mesma forma, será necessário explorar o papel do planejamento por meio da técnica de implementação de intenções, assim como dos processos atencionais.

\section{Referências}

Banaji. M. R., \& Crowder, R. G. (1989). The bankruptcy of everyday memory. American Psychologist, 44, 1185-1193.

Brandimonte, M. A., \& Passolunghi, M. C. (1994). The effect of cue-familiarity, cue-distinctiveness, and retention interval on prospective remembering. The Quarterly Journal of Experimental Psychology, 47, 565-587.

Camp, C. J., Foss, J. W., Stevens, A. B., \& O'Hanlon, A. M. (1996). Memory interventions for persons with dementia. Applied Cognitive Psychology, 10, 193-210.

Craik, F. I. M., \& Lockhart, R. S. (1972). Levels of processing: A framework for memory research. Journal of Verbal Learning and Verbal Behavior, 11, 671-684.

Dismukes, K. (2010). Remembrance of things future: Prospective memory in laboratory, workplace and everyday situations. Reviews of Human Factors and Ergonomics, 6, 79-122.

Dobbs, A. R., \& Rule, B. G. (1987). Prospective memory and selfreports of memory abilities in older adults. Canadian Journal of Psychology, 41, 209-222.

Einstein, G. O., \& McDaniel, M. A (1996). Retrieval processes in prospective memory: Theoretical approaches and some new empirical findings. In M. Bandimonte, G. Einstein, \& M. McDaniel (Eds.), Prospective memory: Theory and applications (pp. 115-142). Hillsdale, NJ: Erlbaum.

Einstein, G. O., \& McDaniel, M. A. (1990). Normal aging and prospective memory. Journal of Experimental Psychology: Learning, Memory, and Cognition, 16, 717-726.

Einstein, G. O., \& McDaniel, M. A. (2005). Prospective memory: Multiple retrieval processes. Current Directions in Psychological Science, 14, 286-290.

Einstein, G. O., Holland, L. J., McDaniel, M. A., \& Guynn, M. J. (1992). Age-related deficits in prospective memory: The influence of task complexity. Psychology and Aging, 7, 471478.

Einstein, G. O., McDaniel, M. A., Smith, R. E., \& Shaw, P. (1998). Habitual prospective memory and aging: Remembering intentions and forgetting actions, Psychological Science, 9, 284-288.

Einstein, G. O., McDaniel, M. A., Thomas, R., Mayfield, S., Shank, H., \& Morrisette, N. (2005). Multiple processes in prospective memory retrieval: Factors determining monitoring versus spontaneous retrieval. Journal of Experimental Psychology: General, 134, 327-342.

Ellis, J. (1996). Prospective memory or the realization of delayed intentions: A conceptual framework for research. In M. Bandimonte, G. Einstein, \& M. McDaniel (Eds.), Prospective memory: Theory and applications (pp. 115-142). Hillsdale, NJ: Erlbaum.

Ellis, J., \& Kvavilashvili, L. (2000). Prospective memory in 2000: Past, present, and future direction. Applied Cognitive Psychology, 14, S1-S9.

Finstad, K., Bink, M., McDaniel, M. A., \& Einstein, G. O. (2006). Breaks and task switches in prospective memory. Applied Cognitive Psychology, 20, 705-712.

Freeman, J. E., \& Ellis, J. A. (2003). The representation of delayed intentions: A prospective subject-performed task? Journal of Experimental Psychology: Learning, Memory, and Cognition, 29, 976-992.

Gollwitzer, P. M. (1999). Implementation intentions: Strong effects of simple plans. American Psychologist, 54, 493-503. 
Goschke, T., \& Kuhl, J. (1993). Representation of intention: Persisting activation in memory. Journal of Experimental Psychology: Learning, Memory, and Cognition, 19, 1211-1226.

Graf, P., \& Uttl, B. (2001). Prospective memory: A new focus of research. Consciousness and Cognition, 10, 437-450.

Guajardo, N. R., \& Best, D. L. (2000). Do preschoolers remember what to do? Incentive and external cues in prospective memory. Cognitive Development, 15, 75-97.

Guynn, M. J. (2003). A two-process model of strategic monitoring in event-based prospective memory: Activation/retrieval mode and checking. International Journal of Psychology, $38,245-256$.

Guynn, M. J., McDaniel, M. A., \& Einstein, G. O. (1998). Prospective memory: When reminders fail. Memory \& Cognition, 26, 287-298.

Harris, J. E. (1984). Remembering to do things: A forgotten topic. In J. E. Harris, \& P. E. Morris (Eds.), Everyday memory, actions, and absent mindedness (pp. 71-92). London: Academic Press.

Hicks, J. L., Marsh, R. L., \& Russell, E. J. (2000). The properties of retention intervals and their affect on retaining prospective memories. Journal of Experimental Psychology: Learning, Memory, and Cognition, 26, 1160-1169.

Kliegel, M., Martin, M., McDaniel, M. A., \& Einstein, G. O. (2002). Complex prospective memory and executive control of working memory: A process model. Psychologische Beiträge, 44, 303-318.

Kliegel, M., Martin, M., McDaniel, M.A., Einstein, G. O., \& Moor, C. (2007). Realizing complex delayed intentions in young and old adults: The role of planning aids. Memory \& Cognition, 35, 1735-1746.

Kliegel, M., McDaniel, M. A., \& Einstein, G. O. (2008). Prospective memory: Cognitive, neuroscience, developmental and applied perspectives. Hillsdale, NJ: Lawrence Erlbaum Associates.

Liu, L. L., \& Park., D. C. (2004). Aging and medical adherence: The use of automatic processes to achieve effortful things. Psychology and Aging, 19, 318-325.

Loukopoulos, L. D., Dismukes, R. K., \& Barshi, I. (2009). The multitasking myth: Handling complexity in real-world operations. Aldershot, United Kingdom: Ashgate.

Marsh, R. L., \& Hicks, J. L. (1998). Event-based prospective memory and executive control of working memory. Journal of Experimental Psychology: Learning, Memory, and Cognition, 24, 336-349.

Marsh, R. L., Hicks, J. L., \& Cook, G. I. (2005). On the relationship between effort toward an ongoing task and cue detection in event-based prospective memory. Journal of Experimental Psychology: Learning, Memory and Cognition, 31, 68-75.

Marsh., R. L., Hicks, J. L., \& Cook., G. I. (2006). Task interference from prospective memory covaries with contextual associations of fulfilling them. Memory \& Cognition, 34, 1037-1045.

Martin, M., Kliegel, M., \& McDaniel, M. A. (2003). The involvement of executive functions in prospective memory performance of adults. International Journal of Psychology, 38, 195-206.

McDaniel, M. A., \& Einstein, G. O. (2000). Strategic and automatic processes in prospective memory retrieval: A multiprocess framework. Applied Cognitive Psychology, 14, 127-144.

McDaniel, M. A., \& Einstein, G. O. (2007). Prospective memory: An overview and synthesis of an emerging field. London: Sage.
McDaniel, M. A., Guynn, M. J., Einstein, G. O., \& Breneiser, J. E. (2004). Cue focused and automatic-associative processes in prospective memory. Journal of Experimental Psychology: Learning. Memory and Cognition, 30, 605-614.

Meacham, J. A., \& Leiman. B. (1982). Remembering to perform future actions. In U. Neisser (Ed.), Memory observed: Remembering in natural contexts (pp. 327-336). San Francisco: Freeman.

Norman, W., \& Shallice, T. (1983). Attention to action. In R. J. Davidson, G. E. Schwartz, \& D. Shapiro (Eds.), Consciousness and self-regulation: Advances in research and theory, Vol. 3 (pp. 1-18). New York: Plenum Press.

Roediger, H.L., III, \& Karpicke, J. D. (2006). Test enhanced learning: Taking memory tests improves long-term retention. Psychological Science, 17, 249-255.

Schank, R. C., \& Abelson, R. P. (1977). Scripts, plans goals and understanding: An enquiry into human knowledge structures. Hillsdale, NJ: Lawrence Erlbaum Associates.

Sellen, A. J., Louie, G., Harris, J. E., \& Wilkins, A. J. (1997). What brings intentions to mind? An in situ study of prospective memory. Memory, 5, 483-507.

Shallice, T., \& Burgess, P. (1991). Deficits in strategy application following frontal lobe damage in man. Brain, 114, 727-741.

Smith, R. E. (2003). The cost of remembering to remember in eventbased prospective memory: Investigating the capacity demands of delayed intention performance. Journal of Experimental Psychology: Learning, Memory and Cognition, 29, 357-361.

Smith, R. E., \& Bayen, U. J. (2004). A multinomial model of event-based prospective memory. Journal of Experimental Psychology: Learning, Memory, and Cognition, 30, 756-777.

Ward, H., Shum, D., McKinlay, L., Baker-Tweney, S., \& Wallace, G. (2005). Development of prospective memory: Tasks based on the prefrontal-lobe model. Child Neuropsychology, 11, 527-549.

West, R. L. (1996) An application of prefrontal cortex theory to cognitive aging. Psychological Bulletin, 120, 272-292.

Zimmermann, T. \& Meier, B. (2010). The effect of implementation intentions on prospective memory performance across the lifespan. Applied Cognitive Pshychology, 24, 645-658.

Recebido em 25.03.2013

Primeira decisão editorial em 31.07.2013

Versão final em 06.08.2013

Aceito em 07.08.2013 